\title{
PARTIAL AUTOMORPHISMS OF STABLE C*-ALGEBRAS AND HILBERT C*-BIMODULES
}

\author{
KAZUNORI KODAKA
}

(Received 10 October 2002; revised 8 December 2004)

Communicated by G. Willis

\begin{abstract}
Let $A$ be a $C^{*}$-algebra and $\mathbb{K}$ the $\mathrm{C}^{*}$-algebra of all compact operators on a countably infinite dimensional Hilbert space. In this note, we shall show that there is an isomorphism of a semigroup of equivalence classes of certain partial automorphisms of $A \otimes \mathbb{K}$ onto a semigroup of equivalence classes of certain countably generated $A$ - $A$-Hilbert bimodules.
\end{abstract}

2000 Mathematics subject classification: primary 46L08; secondary 46L40.

\section{Introduction}

Let $A$ be a $C^{*}$-algebra and $\mathbb{K}$ the $\mathrm{C}^{*}$-algebra of all compact operators on a countably infinite dimensional Hilbert space. Brown, Green and Rieffel [4] defined Pic $(A)$, the Picard group of $A$ as the group of isomorphic classes of $A$ - $A$-equivalence bimodules and showed that there is a homomorphism of $\operatorname{Aut}(A \otimes \mathbb{K})$, the group of all automorphisms of $A \otimes \mathbb{K}$ to $\operatorname{Pic}(A)$ and that its kernel is $\operatorname{Int}(A \otimes \mathbb{K})$, the normal subgroup of $\operatorname{Aut}(A \otimes \mathbb{K})$ of all generalized inner automorphisms of $A \otimes \mathbb{K}$. If $A$ is $\sigma$-unital, the homomorphism of $\operatorname{Aut}(A \otimes \mathbb{K})$ to $\operatorname{Pic}(A)$ is surjective. Hence $\operatorname{Out}(A \otimes \mathbb{K})(=\operatorname{Aut}(A \otimes \mathbb{K}) / \operatorname{Int}(A \otimes \mathbb{K}))$ is isomorphic to $\operatorname{Pic}(A)$.

In this note, we shall give a similar result to the above one for certain partial automorphisms of $A \otimes \mathbb{K}$ and certain countably generated $A$ - $A$-Hilbert bimodules.

For a $C^{*}$-algebra $B$, let $\operatorname{Aut}(B)$ be the group of all automorphisms of $B$ and $M(B)$ its multiplier algebra.

(C) 2005 Australian Mathematical Society $1446-7887 / 05 \$ A 2.00+0.00$ 


\section{Equivalence relations and a map}

Let $A$ and $\mathbb{K}$ be as above. Let $\operatorname{PAut}(A \otimes \mathbb{K})$ be the set of all partial automorphisms of $A \otimes \mathbb{K}$ defined by Exel [6]. We shall give an equivalence relation $\sim$ as follows; for $\Theta_{j}=\left(I_{j}, J_{j}, \theta_{j}\right) \in \operatorname{PAut}(A \otimes \mathbb{K})(j=1,2), \Theta_{1} \sim \Theta_{2}$ if $I_{1}=I_{2}, J_{1}=J_{2}$ and there is a unitary element $w \in M\left(I_{1}\right)$ such that $\theta_{2}=\theta_{1} \circ \operatorname{Ad}(w)$. We denote by $[\Theta]$ the equivalence class of a partial automorphism $\Theta \in \operatorname{PAut}(A \otimes \mathbb{K})$. We also denote by $\operatorname{PAut}(A \otimes \mathbb{K}) / \sim$ the quotient set of $\operatorname{PAut}(A \otimes \mathbb{K})$ by the above equivalence relation. We shall define a product in $\operatorname{PAut}(A \otimes \mathbb{K}) / \sim$ as follows. Let $\Theta_{j}=\left(I_{j}, J_{j}, \theta_{j}\right) \in$ $\operatorname{PAut}(A \otimes \mathbb{K})(j=1,2)$, and let $I_{3}=\theta_{1}^{-1}\left(J_{1} \cap I_{2}\right), J_{3}=\theta_{2}\left(J_{1} \cap I_{2}\right), \theta_{3}=\theta_{2} \circ \theta_{1}$. Finally, let $\Theta_{3}=\left(I_{3}, J_{3}, \theta_{3}\right) \in \operatorname{PAut}(A \otimes \mathbb{K})$. We define $\left[\Theta_{1}\right]\left[\Theta_{2}\right]=\left[\Theta_{3}\right]$. By routine computations, we can see that $\left[\Theta_{3}\right]$ is independent of the choices of representatives of $\left[\Theta_{1}\right]$ and $\left[\Theta_{2}\right]$. By the above product, $\operatorname{PAut}(A \otimes \mathbb{K}) / \sim$ is a semigroup. Let $\Theta_{e}=\left(A \otimes \mathbb{K}, A \otimes \mathbb{K}, i d_{A \otimes \mathbb{K}}\right) \in \operatorname{PAut}(A \otimes \mathbb{K})$. Then by easy computations [ $\left.\Theta_{e}\right]$ is the unit element in $\operatorname{PAut}(A \otimes \mathbb{K}) / \sim$ and the group of all invertible elements in $\operatorname{PAut}(A \otimes \mathbb{K}) / \sim$ is

$$
\{[(A \otimes \mathbb{K}, A \otimes \mathbb{K}, \beta)] \mid \beta \in \operatorname{Aut}(A \otimes \mathbb{K})\} \cong \operatorname{Out}(A \otimes \mathbb{K}) .
$$

We identify it with $\operatorname{Out}(A \otimes \mathbb{K})$ and denote an element $[(A \otimes \mathbb{K}, A \otimes \mathbb{K}, \beta)] \in$ $\operatorname{PAut}(A \otimes \mathbb{K}) / \sim$ by $[B]$.

Let $\operatorname{HB}(A)$ be the set of all $A$ - $A$-Hilbert bimodule isomorphic classes of $A-A$ Hilbert bimodules defined in Brown, Mingo and Shen [5] and Abadie, Eilers and Exel [1]. For any $A$ - $A$-Hilbert bimodule $X$, we denote by $[X]$ the $A$ - $A$-Hilbert bimodule isomorphic class of $X$. We define a product in $\operatorname{HB}(A)$ as the relative tensor product with respect to $A$.

In the same way as in Abadie, Eilers and Exel [1, Example 3.2], for any $\Theta=$ $(I, J, \theta) \in \operatorname{PAut}(A \otimes \mathbb{K})$ we define an $A \otimes \mathbb{K}-A \otimes \mathbb{K}$-Hilbert bimodule $X_{\Theta}$ as follows. Let $X_{\Theta}$ be the vector space $I$ and the obvious left action of $A \otimes \mathbb{K}$ and the obvious left inner product, but we define the right action of $A \otimes \mathbb{K}$ on $X_{\Theta}$ by $x \cdot a=\theta^{-1}(\theta(x) a)$ for any $a \in A \otimes \mathbb{K}$ and $x \in X_{\Theta}$ and the right $A \otimes \mathbb{K}$-valued inner product by $\langle x, y\rangle_{A \otimes \mathbb{K}}=\theta\left(x^{*} y\right)$ for any $x, y \in X_{\Theta}$.

LEMma 1.1. Let $\Theta_{j}=\left(I_{j}, J_{j}, \theta_{j}\right) \in \operatorname{PAut}(A \otimes \mathbb{K})(j=1,2)$. If $\Theta_{1} \sim \Theta_{2}$, then $X_{\Theta_{1}} \cong X_{\Theta_{2}}$ as $A \otimes \mathbb{K}-A \otimes \mathbb{K}$-Hilbert bimodules.

Proof. We shall prove this lemma in the same way as in Brown, Green and Rieffel [4, Proposition 3.1]. Since $\Theta_{1} \sim \Theta_{2}, I_{1}=I_{2}$ and $J_{1}=J_{2}$. Put $I=I_{1}=I_{2}$ and $J=J_{1}=J_{2}$. Then there is a unitary element $w \in M(I)$ such that $\theta_{2}=\theta_{1} \circ \operatorname{Ad}(w)$. Let $\Phi$ be a map from $X_{(-)_{1}}$ to $X_{()_{2}}$ defined by $\Phi(x)=x w$ for any $x \in X_{()_{1}}$. Then by direct 
computations, we can see that $\Phi$ is an $A \otimes \mathbb{K}-A \otimes \mathbb{K}$ - Hilbert bimodule isomorphism of $X_{\Theta_{1}}$ onto $X_{\Theta_{2}}$ since $\theta_{2}=\theta_{1} \circ \operatorname{Ad}(w)$. Hence we obtain the conclusion.

By Lemma 1.1, the map $[\Theta] \in \operatorname{PAut}(A \otimes \mathbb{K}) / \sim \rightarrow\left[X_{\Theta}\right] \in \mathrm{HB}(A \otimes \mathbb{K})$ can be defined.

LeMma 1.2. The map $[\Theta] \in \operatorname{PAut}(A \otimes \mathbb{K}) / \sim \rightarrow\left[X_{\Theta}\right] \in \mathrm{HB}(A \otimes \mathbb{K})$ is $a$ semigroup homomorphism.

PROOF. Let $\Theta_{j}=\left(I_{j}, J_{j}, \theta_{j}\right) \in \operatorname{PAut}(A \otimes \mathbb{K})(j=1,2)$. Let $\Theta_{3}=\left(\theta_{1}^{-1}\left(J_{1} \cap\right.\right.$ $\left.\left.I_{2}\right), \theta_{2}\left(J_{1} \cap I_{2}\right), \theta_{2} \circ \theta_{1}\right) \in \operatorname{PAut}(A \otimes \mathbb{K})$. Then by the definition of the product in $\operatorname{PAut}(A \otimes \mathbb{K}) / \sim,\left[\Theta_{3}\right]=\left[\Theta_{1}\right]\left[\Theta_{2}\right]$. Hence it suffices to show that $X_{\Theta_{1}} \otimes_{A \otimes \mathbb{K}} X_{\Theta_{2}} \cong$ $X_{\Theta_{3}}$ as $A \otimes \mathbb{K}-A \otimes \mathbb{K}$-Hilbert bimodules. We note that $X_{\Theta_{1}}=I_{1}, X_{\Theta_{2}}=I_{2}$ and $X_{\Theta_{3}}=\theta_{1}^{-1}\left(J_{1} \cap I_{2}\right)$ as vector spaces. Let $\Phi$ be a map of $X_{\Theta_{1}} \otimes_{A \otimes \mathbb{K}} X_{\Theta_{2}}$ to $X_{\Theta_{3}}$ defined by $\Phi\left(x_{1} \otimes x_{2}\right)=\theta_{1}^{-1}\left(\theta_{1}\left(x_{1}\right) x_{2}\right)$ for any $x_{1} \in X_{\Theta_{1}}$ and $x_{2} \in X_{\Theta_{2}}$. For any $a \in A \otimes \mathbb{K}$ and $x_{1} \in X_{\Theta_{1}}, x_{2} \in X_{\Theta_{2}}$ with $x_{1}, x_{2} \geq 0$,

$$
\Phi\left(x_{1} \otimes\left(a \cdot x_{2}\right)\right)=\theta_{1}^{-1}\left(\theta_{1}\left(x_{1}\right) a x_{2}\right)=\Phi\left(\left(x_{1} \cdot a\right) \otimes x_{2}\right) .
$$

Thus $\Phi$ is well defined. It is also clear that $\Phi$ is surjective. Furthermore, by routine computations, $\Phi$ preserves the both inner products. Hence, by the remark after Jensen and Thomsen [7, Definition 1.1.18], $X_{\Theta_{1}} \otimes_{A \otimes \mathbb{K}} X_{\Theta_{2}} \cong X_{\Theta_{3}}$ as $A \otimes \mathbb{K}-A \otimes \mathbb{K}$-Hilbert bimodules. Therefore, we obtain the conclusion.

For any $A$-A-Hilbert bimodule, let $I_{L}(X)$ be the closure of linear span of $\left.\right|_{A}\langle x, y\rangle \mid$ $x, y \in X\}$ and $I_{R}(X)$ the closure of linear span of $\left\{\langle x, y\rangle_{A} \mid x, y \in X\right\}$. By Brown, Mingo and Shen [5, Remark 1.9], $I_{L}(X)$ and $I_{R}(X)$ are closed two-sided ideals of $A$ and by restriction we regard $X$ as an $I_{L}(X)-I_{R}(X)$-equivalence bimodule.

LEMMA 1.3. The homomorphism $[\Theta] \in \operatorname{PAut}(A \otimes \mathbb{K}) / \sim \rightarrow\left[X_{\Theta}\right] \in \mathrm{HB}(A \otimes \mathbb{K})$ is injective.

Proof. Let $\Theta_{j} \in \operatorname{PAut}(A \otimes \mathbb{K})(j=1,2)$. We suppose that $X_{\Theta_{1}} \cong X_{\Theta_{2}}$ as $A \otimes \mathbb{K}-A \otimes \mathbb{K}$-Hilbert bimodules. Thus there is an $A \otimes \mathbb{K}-A \otimes \mathbb{K}$-Hilbert bimodule isomorphism $\Phi$ of $X_{\Theta_{1}}$ onto $X_{\Theta_{2}}$. Then since $\Phi$ preserves the both inner products, $I_{L}\left(X_{\Theta_{1}}\right)=I_{L}\left(X_{\Theta_{2}}\right)$ and $I_{R}\left(X_{\Theta_{1}}\right)=I_{R}\left(X_{\Theta_{2}}\right)$. On the other hand, $I_{L}\left(X_{\Theta_{1}}\right)=I_{j}$, $I_{R}\left(X_{\Theta_{j}}\right)=J_{j}$ by the definition of $X_{\Theta_{j}}$ for $j=1,2$. Thus $I_{1}=I_{2}$ and $J_{1}=J_{2}$. We regard $X_{\Theta_{1}}$ and $X_{\Theta_{2}}$ as $I-J$-equivalence bimodules where $I=I_{1}=I_{2}$ and $J=J_{1}=J_{2}$. Then $X_{\Theta_{1}} \cong X_{\Theta_{2}}$ as $I$ - $J$-equivalence bimodules. Hence, by Brown, Green and Rieffel [4, Corollary 3.2], there is a unitary element $w \in M(I)$ such that $\theta_{2}=\theta_{1} \circ \operatorname{Ad}(w)$. Therefore, $\Theta_{1} \sim \Theta_{2}$. 
Let $B$ be a $C^{*}$-algebra. For each $A$ - $B$-Hilbert bimodule $X$, let $\tilde{X}$ be its dual Hilbert $\mathrm{C}^{*}$-bimodule. Let $\mathbb{H}_{A}$ be the standard equivalence bimodule between $A \otimes \mathbb{K}$ and $A$, that is, $\mathbb{H}_{A}=(A \otimes \mathbb{K})(1 \otimes e)$, where 1 is the unit element in $M(A), e$ is a rank one projection in $\mathbb{K}$ and we identify $A$ with $A \otimes e$. For any $\Theta \in \operatorname{PAut}(A \otimes \mathbb{K})$, let $Y_{\Theta}=\tilde{\mathbb{T}}_{A} \otimes_{A \otimes K} X_{\Theta} \otimes_{A \otimes K} \mathbb{H}_{A}$. Then the map $[X] \rightarrow\left[\tilde{\mathbb{T}}_{A} \otimes_{A \otimes K} X \otimes_{A \otimes K} \mathbb{H}_{A}\right]$ is an isomorphism of $\mathrm{HB}(A \otimes \mathbb{K})$ onto $\mathrm{HB}(A)$. Thus the map $[\Theta] \in \operatorname{PAut}(A \otimes \mathbb{K}) / \sim \rightarrow$ $\left[Y_{\Theta}\right] \in \mathrm{HB}(A)$ is injective.

COROLlaRY 1.4. The map $[\Theta] \in \operatorname{PAut}(A \otimes \mathbb{K}) / \sim \rightarrow\left[Y_{\Theta}\right] \in \mathrm{HB}(A)$ is an injective homomorphism.

\section{A certain class of countably generated Hilbert bimodules and a certain class of partial automorphisms}

Let $Y$ be an $A$-A-Hilbert bimodule. We say that $Y$ is countably generated as equivalence bimodules if $Y$ is a countably generated left Hilbert $I_{L}(Y)$-module and $Y$ is a countably generated right Hilbert $I_{R}(Y)$-module.

Let $Y$ be an $A$ - $A$-Hilbert bimodule countably generated as equivalence bimodules. By Blackadar [2, Corollary 13.6.3] $I_{L}(Y)$ and $I_{R}(Y)$ are $\sigma$-unital. Put $I=I_{L}(Y) \otimes \mathbb{K}$, $J=I_{R}(Y) \otimes \mathbb{K}$. Then $I$ and $J$ are also $\sigma$-unital by Brown, Green and Rieffel [4, Proposition 2.4]. Let $X=\mathbb{H}_{A} \otimes_{A} Y \otimes_{A} \tilde{\mathbb{H}}_{A}$. Then

$$
\begin{aligned}
& I_{L}(X)=\overline{{ }_{A \otimes K}\left\langle\mathbb{H}_{A A}\left\langle Y_{A}\left\langle\tilde{\mathbb{H}}_{A}, \tilde{\mathbb{H}}{ }_{A}\right\rangle, Y\right\rangle, \mathbb{H}_{A}\right\rangle}
\end{aligned}
$$

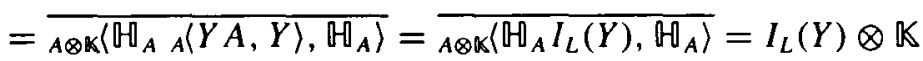

since $\overline{Y A}=Y$ by Brown, Mingo and Shen [5, Proposition 1.7]. Similarly $I_{R}(X)=$ $I_{R}(Y) \otimes \mathbb{K}$.

LEMMA 2.1. Let $Y$ be an A-A-Hilbert bimodule countably generated as equivalence bimodules. Then there is a partial automorphism $\Theta_{Y}$ of $A \otimes \mathbb{K}$ such that $Y_{\Theta_{Y}} \cong Y$ as A-A-Hilbert bimodules.

Proof. Let $I$ and $J$ be as above. Then $I$ and $J$ are $\sigma$-unital. Hence, by Brown, Green and Rieffel [4, Theorem 3.4], there is an isomorphism $\theta$ of $I$ onto $J$ such that $X_{\theta} \cong X$ as $I$ - $J$-equivalence bimodules where $X_{\theta}$ is an $I$ - $J$-equivalence bimodule induced by the isomorphism $\theta$ of $I$ onto $J$ which is defined in Brown, Green and Rieffel [4, Section 3] and $X=\mathbb{H}_{A} \otimes_{A} Y \otimes_{A} \tilde{\mathbb{H}}_{A}$. Let $\Theta_{Y}$ be a partial automorphism of $A \otimes \mathbb{K}$ defined by $\Theta_{Y}=(I, J, \theta)$. Then by the definition of $X_{\Theta_{Y}}, X_{\Theta_{Y}}=X_{\theta}$ as $I$ - $J$-equivalence bimodules. Hence $X_{\Theta_{Y}} \cong X$ as $I-J$-equivalence bimodules. By the remark after Jensen and Thomsen [7, Definition 1.1.18], $X_{\Theta_{r}} \cong X$ as $A \otimes \mathbb{K}-A \otimes \mathbb{K}$ Hilbert bimodules. Therefore, $Y_{\Theta_{Y}} \cong Y$ as $A$ - $A$-Hilbert bimodules. 
Let $\mathrm{PAut}_{\sigma}(A \otimes \mathbb{K})$ be the set of all partial automorphisms $\Theta=(I, J, \theta)$ of $A \otimes \mathbb{K}$ satisfying that $I$ and $J$ are $\sigma$-unital. Let $\operatorname{HB}_{\mathrm{c}}(A)$ be the subsemigroup of all $A$ - $A$ Hilbert bimodules countably generated as equivalence bimodules.

LEMMA 2.2. $\mathrm{HB}_{\mathrm{c}}(A)$ is the image of a subsemigroup $\mathrm{PAut}_{\sigma}(A \otimes \mathbb{K}) / \sim$ by the homomorphism $[\Theta] \in \operatorname{PAut}(A \otimes \mathbb{K}) / \sim \rightarrow\left[Y_{\Theta}\right] \in \mathrm{HB}(A)$.

PROOF. By the proof of Lemma 2.1, we see that $\mathrm{HB}_{\mathrm{c}}(A)$ is contained in the image of $\operatorname{PAut}_{\sigma}(A \otimes \mathbb{K}) / \sim$ by the map $[\Theta] \in \operatorname{PAut}(A \otimes \mathbb{K}) / \sim \rightarrow\left[Y_{\Theta}\right] \in \mathrm{HB}(A)$. Hence we have only to show the inverse inclusion. Let $\Theta=(I, J, \theta) \in$ PAut $_{\sigma}(A \otimes \mathbb{K})$. Then since $\overline{X_{\Theta}(A \otimes \mathbb{K})}=X_{\Theta}$ by Brown, Mingo and Shen [5, Proposition 1.7],

$$
\begin{aligned}
& I_{L}\left(Y_{\Theta}\right)=\overline{{ }_{A}\left\langle\tilde{\mathbb{H}}_{A A \otimes K}\left\langle X_{\Theta A \otimes K}\left\langle\mathbb{H}_{A}, \mathbb{H}_{A}\right\rangle, X_{\Theta}\right\rangle, \tilde{\mathbb{H}}_{A}\right\rangle} \\
& =\overline{{ }_{A}\left(\tilde{\mathbb{Q}}_{A}{ }_{A \otimes K}\left\langle X_{\Theta}, X_{\Theta}\right\rangle, \tilde{\mathbb{V}}_{A}\right\rangle}=\overline{\left\langle I \mathbb{H}_{A}, \mathbb{H}_{A}\right\rangle_{A}} \\
& =(1 \otimes e) I(1 \otimes e) \text {. }
\end{aligned}
$$

Let $(A \otimes \mathbb{K})^{\prime \prime}$ and $I^{\prime \prime}$ be the universal von Neumann algebras of $A \otimes \mathbb{K}$ and $I$, respectively. Then by Pedersen [8], there is a central open projection $p \in(A \otimes \mathbb{K})^{\prime \prime}$ with $I^{\prime \prime}=p(A \otimes \mathbb{K})^{\prime \prime}$ and $I=p(A \otimes \mathbb{K})^{\prime \prime} \cap(A \otimes \mathbb{K})$. Put $q=(1 \otimes e) p$. Then $q$ is a full projection in $M(I)$ and $I_{L}\left(Y_{\Theta}\right)=q I q$. Since $I$ is $\sigma$-unital, by Brown [3, Corollary 2.6], $I_{L}\left(Y_{\Theta}\right)$ is stably isomorphic to $I$. Hence, by Brown, Green and Rieffel [4, Proposition 2.4] $I_{L}\left(Y_{\Theta}\right)$ is $\sigma$-unital since $I$ is $\sigma$-unital. Similarly, $I_{R}\left(Y_{\Theta}\right)$ is also $\sigma$-unital. Thus, by Blackadar [2, Corollary 13.6.3], $Y_{\Theta}$ is countably generated as equivalence bimodules. Therefore, $\left[Y_{\Theta}\right] \in \mathrm{HB}_{\mathrm{c}}(A)$.

By Corollary 1.4 and Lemma 2.2, we obtain the following theorem.

THEOREM 2.3. The map $[\Theta] \rightarrow\left[Y_{\Theta}\right]$ is an isomorphism of PAut $_{\sigma}(A \otimes \mathbb{K}) / \sim$ onto $\mathrm{HB}_{\mathrm{c}}(A)$.

Using the proof of Lemma 2.1, we obtain the following corollary.

COROLLARY 2.4. For any $\sigma$-unital closed two-sided ideal I of $A \otimes \mathbb{K}$, there is a $\sigma$-unital closed two-sided ideal $1_{0}$ of $A$ such that $I \cong I_{0} \otimes \mathbb{K}$.

\section{Conjugacy classes}

For a $\mathrm{C}^{*}$-algebra $A$, let $\mathrm{HB}(A)^{-1}$ be the set of all invertible elements in $\mathrm{HB}(A)$. Then $\operatorname{Pic}(A)=\operatorname{HB}(A)^{-1}$. We consider the set of conjugacy classes of elements in $\mathrm{HB}(A)$ by an invertible element in $\operatorname{HB}(A)$. That is, for any $\left[X_{1}\right],\left[X_{2}\right] \in \mathrm{HB}(A),\left[X_{1}\right]$ 
is equivalent to $\left[X_{2}\right]$, written $\left[X_{1}\right] \sim\left[X_{2}\right]$, if there is an element $[Y] \in \operatorname{Pic}(A)$ such that $\left[X_{2}\right]=[Y]\left[X_{1}\right][Y]^{-1}$. We denote by $\operatorname{HB}(A) / \sim$ the quotient set by the above equivalence relation and denote by $[[X]]$ the equivalence class of $[X] \in \mathrm{HB}(A)$. By Brown, Green and Rieffel [4, Proposition 3.1], we can easily consider the following conjecture:

CONJECTURE. There is a map from $\operatorname{PAut}(A \otimes \mathbb{K}) / \approx$ to $\mathrm{HB}(A) / \sim$ induced by the homomorphism of $\operatorname{PAut}(A \otimes \mathbb{K}) / \sim$ to $\mathrm{HB}(A)$ defined in Section 2 if we give an appropriate equivalence relation $\sim$ in $\operatorname{PAut}(A \otimes \mathbb{K}) / \sim$, where $\operatorname{PAut}(A \otimes \mathbb{K}) / \approx$ is the quotient set of $\mathrm{PAut}(A \otimes \mathbb{K}) / \sim$ by the appropriate equivalence relation. Furthermore, the map from $\operatorname{PAut}(A \otimes \mathbb{K}) / \approx$ to $\mathrm{HB}(A) / \sim$ is injective if $A$ is $\sigma$-unital.

In this section, we shall show this conjecture and obtain the main theorem. First, we shall give an equivalence relation $\sim$ in $\operatorname{PAut}(A \otimes \mathbb{K}) / \sim$ as follows; for $\Theta_{j}=$ $\left(I_{j}, J_{j}, \theta_{j}\right) \in \operatorname{PAut}(A \otimes \mathbb{K})(j=1,2),\left[\Theta_{1}\right] \sim\left[\Theta_{2}\right]$ if there is a $\beta \in \operatorname{Aut}(A \otimes \mathbb{K})$ such that $I_{2}=\beta\left(I_{1}\right), J_{2}=\beta\left(J_{1}\right)$ and $\left[\Theta_{1}\right]=\left[\left(I_{1}, J_{1}, \beta^{-1} \circ \theta_{2} \circ \beta\right)\right]$. We denote by $[[\Theta]]$ the equivalence class of $[\Theta] \in \operatorname{PAut}(A \otimes \mathbb{K}) / \sim$.

LEMMA 3.1. Let $\Theta_{j}=\left(I_{j}, J_{j}, \theta_{j}\right) \in \operatorname{PAut}(A \otimes \mathbb{K})$ for $j=1$, 2. If $\left[\Theta_{1}\right] \sim\left[\Theta_{2}\right]$, $\left[Y_{\Theta_{1}}\right] \sim\left[Y_{\Theta_{2}}\right]$.

Proof. Since $\left[\Theta_{1}\right] \sim\left[\Theta_{2}\right]$, there is a $\beta \in \operatorname{Aut}(A \otimes \mathbb{K})$ such that $I_{2}=\beta\left(I_{1}\right)$, $J_{2}=\beta\left(J_{1}\right)$ and $\left[\Theta_{1}\right]=\left[\left(I_{1}, J_{1}, \beta^{-1} \circ \theta_{2} \circ \beta\right)\right]$. By the definition of the product in $\operatorname{PAut}(A \otimes \mathbb{K}) / \sim$,

$$
\begin{aligned}
{\left[\Theta_{1}\right] } & =\left[\left(I_{1}, J_{1}, \beta^{-1} \circ \theta_{2} \circ \beta\right)\right] \\
& =[(A \otimes \mathbb{K}, A \otimes \mathbb{K}, \beta)]\left[\Theta_{2}\right]\left[\left(A \otimes \mathbb{K}, A \otimes \mathbb{K}, \beta^{-1}\right)\right]=[\beta]\left[\Theta_{2}\right][\beta]^{-1} .
\end{aligned}
$$

Thus using the homomorphism of $\operatorname{PAut}(A \otimes \mathbb{K}) / \sim$ to $\mathrm{HB}(A)$ defined in Section 2, we see that $\left[Y_{\Theta_{1}}\right]=\left[Y_{\beta}\right]\left[Y_{\Theta_{2}}\right]\left[Y_{\beta}\right]^{-1}$, where $Y_{\beta}=\tilde{\mathbb{H}}_{A} \otimes_{A \otimes K} X_{\beta} \otimes_{A \otimes K} \mathbb{H}_{A}$ and $X_{\beta}$ is an $A \otimes \mathbb{K}-A \otimes \mathbb{K}$-equivalence bimodule induced by $\beta$, which is defined in Brown, Green and Rieffel [4, Section 3]. Therefore, we obtain the conclusion.

By Lemma 3.1, we can define a map

$$
[[\Theta]] \in \operatorname{PAut}(A \otimes \mathbb{K}) / \approx \rightarrow\left[\left[Y_{\Theta}\right]\right] \in \mathrm{HB}(A) / \sim .
$$

LEMMA 3.2. If $A$ is a $\sigma$-unital $C^{*}$-algebra, the map (1) is injective.

PROOF. Let $\Theta_{j}=\left(I_{j}, J_{j}, \theta_{j}\right) \in \operatorname{PAut}(A \otimes \mathbb{K})(j=1,2)$ with $\left[Y_{\Theta_{1}}\right] \sim\left[Y_{\Theta_{2}}\right]$. Since $A$ is $\sigma$-unital, by Brown, Green and Rieffel [4, Corollary 3.5], there is a $\beta \in \operatorname{Aut}(A \otimes \mathbb{K})$ such that $\left[Y_{\Theta_{1}}\right]=\left[Y_{\beta}\right]\left[Y_{\Theta_{-}}\right]\left[Y_{\beta}\right]^{-1}$ where $Y_{\beta}$ is an $A$-A-equivalence bimodule defined in the proof of Lemma 3.1. Since the homomorphism $[\Theta] \in \operatorname{PAut}(A \otimes \mathbb{K}) / \sim \rightarrow$ $\left[Y_{\Theta}\right] \in \mathrm{HB}(A)$ is injective, we obtain that $\left[\Theta_{1}\right]=[\beta]\left[\Theta_{2}\right][\beta]^{-1}$. 
THEOREM 3.3. Let $A$ be a $\sigma$-unital $C^{*}$-algebra. Then the map

$$
[[\Theta]] \in \operatorname{PAut}_{\sigma}(A \otimes \mathbb{K}) / \approx \rightarrow\left[\left[Y_{\Theta}\right]\right] \in \operatorname{HB}_{\mathrm{c}}(A) / \sim
$$

is a bijection.

PROOF. The result follows immediately from Theorem 2.3 and Lemma 3.2.

\section{Crossed products}

In this section, we shall consider a crossed product of a $\mathrm{C}^{*}$-algebra by a Hilbert $\mathrm{C}^{*}$-bimodule defined in Abadie, Eilers and Exel [1].

Let $A$ be a $C^{*}$-algebra and $Y$ an $A$ - $A$-Hilbert bimodule countably generated as equivalence bimodules. Then by Abadie, Eilers and Exel [1, Remark 3.4],

$$
\left(A \times_{Y} \mathbb{Z}\right) \otimes \mathbb{K} \cong(A \otimes \mathbb{K}) \times_{\Theta_{Y}} \mathbb{Z},
$$

where $(A \otimes \mathbb{K}) \times_{\Theta_{Y}} \mathbb{Z}$ is the crossed product of $A \otimes \mathbb{K}$ by $\Theta_{Y}$ which is defined in Exel [6] and $\Theta_{Y}$ is a partial automorphism of $A \otimes \mathbb{K}$ induced by $Y$ which is defined in Section 2. Hence we obtain the following proposition.

PROPOSITION 4.1. Let A be a $C^{*}$-algebra and $Y$ an A-A-Hilbert bimodule countably generated as equivalence bimodules. Then

$$
A \times_{Y} \mathbb{Z} \cong(1 \otimes e)\left((A \otimes \mathbb{K}) \times_{\Theta_{Y}} \mathbb{Z}\right)(1 \otimes e),
$$

where $\Theta_{Y}$ is the partial automorphism of $A \otimes \mathbb{K}$ induced by $Y$ and 1 is the unit element in $M(A), e$ is a rank one projection in $\mathbb{K}$. In particular, if $A$ is a simple $\sigma$-unital $C^{*}$-algebra, there is a $\beta \in \operatorname{Aut}(A \otimes \mathbb{K})$ which is uniquely determined up to multiplication by a generalized inner automorphism of $A \otimes \mathbb{K}$ such that

$$
A \times_{Y} \mathbb{Z} \cong(1 \otimes e)\left((A \otimes \mathbb{K}) \times_{\beta} \mathbb{Z}\right)(1 \otimes e) .
$$

COROLLARY 4.2. Let A be a simple $\sigma$-unital $C^{*}$-algebra and $Y$ an A-A-equivalence bimodule. Then $A \times_{Y} \mathbb{Z}$ is simple if and only if for any $n \in \mathbb{Z} \backslash\{0\},[Y]^{n} \neq[A]$ in $\operatorname{Pic}(A)$.

ProOF. Let $\beta$ be an automorphism of $A \otimes \mathbb{K}$ such that

$$
A \times_{Y} \mathbb{Z} \cong(1 \otimes e)\left((A \otimes \mathbb{K}) \times_{\beta} \mathbb{Z}\right)(1 \otimes e) .
$$

Hence $A \times_{\beta} \mathbb{Z}$ is simple if and only if $\beta^{n} \notin \operatorname{Int}(A \otimes \mathbb{K})$ for any $n \in \mathbb{Z} \backslash\{0\}$ by Pedersen [8, Corollary 8.9.10]. By the proof of Lemma 2.1, $Y \cong \tilde{\mathbb{H}}_{A} \otimes_{A \otimes K} X_{\beta} \otimes_{A \otimes K} \mathbb{H}_{A}$ where $X_{\beta}$ is the $A \otimes \mathbb{K}-A \otimes \mathbb{K}$-equivalence bimodule induced by $\beta$. Therefore, by Brown, Green and Rieffel [4, Corollary 3.5], we obtain the conclusion. 


\section{References}

[1] B. Abadie, S. Eilers and R. Exel, 'Morita equivalence for crossed products by Hilbert C*-bimodules', Trans. Amer. Math. Soc. 350 (1998), 3043-3054.

[2] B. Blackadar, $K$-theory for operator algebras, Mathematical Sciences Research Institute Publications 5, 2nd Edition (Cambridge Univ. Press, Cambridge, 1998).

[3] L. G. Brown, 'Stable isomorphism of hereditary subalgebras of C*-algebras', Pacific J. Math. 71 (1977), 335-348.

[4] L. G. Brown, P. Green and M. A. Rieffel, 'Stable isomorphism and strong Morita equivalence of C*-algebras', Pacific J. Math. 71 (1977), 349-363.

[5] L. G. Brown, J. A. Mingo and N. Shen, 'Quasi-multipliers and embeddings of Hilbert C*-bimodules'. Canad. J. Math. 46 (1994), 1150-1174.

[6] R. Exel, "Circle actions on $\mathrm{C}^{*}$-algebras, partial automorphisms and a generalized PimznerVoiculescu exact sequence', J. Funct. Anal. 122 (1994), 361-401.

[7] K. K. Jensen and K. Thomsen, Elements of KK-theory (Birkäuser, 1991).

[8] G. K. Pedersen, $C^{*}$-algebras and their automorphism groups (Academic Press, 1979).

\section{Department of Mathematical Sciences}

Faculty of Science

Ryukyu University Nishihara-cho

Okinawa 903-0213

Japan

e-mail: kodaka@math.u-ryukyu.ac.jp 